\title{
La communauté des Égaux. Le communisme néo- babouviste dans la France des années 1840
}

\section{Claude Mazauric}

\section{(2) OpenEdition}

1 Journals

\section{Édition électronique}

URL : https://journals.openedition.org/ahrf/1007

DOI : 10.4000/ahrf.1007

ISSN : 1952-403X

Éditeur :

Armand Colin, Société des études robespierristes

\section{Édition imprimée}

Date de publication : 1 décembre 2000

Pagination : 161-162

ISSN : 0003-4436

\section{Référence électronique}

Claude Mazauric, «La communauté des Égaux. Le communisme néo-babouviste dans la France des années 1840 ", Annales historiques de la Révolution française [En ligne], 322 I octobre-décembre 2000, mis en ligne le 26 avril 2006, consulté le 23 avril 2022. URL : http://journals.openedition.org/ahrf/1007 ; DOI : https://doi.org/10.4000/ahrf.1007

Ce document a été généré automatiquement le 23 avril 2022.

Tous droits réservés 


\title{
La communauté des Égaux. Le communisme néo-babouviste dans la France des années 1840
}

\author{
Claude Mazauric
}

\section{RÉFÉRENCE}

Alain Maillard, La communauté des Égaux. Le communisme néo-babouviste dans la France des années 1840, Paris, Kimé, 1999, 323 p.+ annexes, bibliographie et index (29 p.), $195 \mathrm{~F}$.

1 Alain Maillard est sociologue à l'université de Picardie - Jules Verne à Amiens. L'ouvrage dont il est ici question trouve son origine dans la thèse de doctorat de l'auteur, préparée sous la direction de Pierre Ansart, éminent spécialiste de la pensée sociale $\mathrm{du} \mathrm{xIX}^{\mathrm{e}}$ siècle belle thèse, beau livre et qui plus est utile synthèse qu'il sera désormais impossible d'ignorer, à l'égal de la somme analytique et documentaire, naguère proposée par Jacques Granjonc (1989) auquel d'ailleurs Alain Maillard dit sa dette dès la page 2. Comme ses prédécesseurs sur ce chantier (Galante-Garrone, Saïtta, Lehning, Dautry, Bruhat, Moissonnier et quelques autres dont moi-même, tous soigneusement étudiés et cités), A. Maillard a eu recours aux deux types de sources qui s'imposent à quiconque s'intéresse au communisme français d'avant 1848 les actes des procès politiques, notamment des procès en régicide intentés devant la Cour des Pairs de la monarchie de Juillet à la suite des attentats dirigés contre Louis-Philippe immenses enquêtes qui conduisent à mettre à jour des réseaux républicains radicaux au sein desquels s'activent les militants disciples de Babeuf et de Buonarroti, entre autres-, d'autre part les journaux, textes imprimés, opuscules, libellés, essais, programmes et protocoles, publiés ou saisis par la police, clandestins ou publics. Au total, dans le corpus de Maillard, huit périodiques parisiens ou lyonnais ( $c f$. la liste pp. 331-332) et plusieurs dizaines d'opéras dont le nom des auteurs est bien connu Benoît, Dezamy, La Hautière, Pillot, Savary etc. , souvent republiés par Édhis, sans compter les collatéraux, adversaires ou non, de Laponneraye à Esquiros ou Cabet. L'enquête de fond 
m'a donc paru, sinon exhaustive - le pourrait-elle - du moins excellemment conduite et de nature à donner force et vigueur à l'énonciation des hypothèses.

2 La première partie de l'ouvrage («Pratiques, structures sociales et communismes égalitaires ») consacre ses cent pages à un retour critique sur les acquis des travaux antérieurs relatifs à l'histoire du communisme, des utopies sociales prérévolutionnaires, retour toujours attentif à rendre compte des configurations sociales, professionnelles et idéologiques qui encadrent l'apparition du néo-babouvisme après 1830 , en réalité après 1832 . La seconde partie (124 pages) concentre l'analyse sur la «vision communiste des années 1840 » dans son rapport de mémoire et d'intelligence au double héritage de la démocratie robespierriste et du "babouvisme » tel que Buonarroti en a proposé l'épure. Fondée sur l'ambition d'une "science sociale »- comme le voulait d'autre manière la philanthropie sous la monarchie de Juillet ( $c f$. la thèse de Catherine Duprat) - l'utopie dès lors, se moule en «science doctrinaire » (p. 214). Ainsi passe-t-on à une troisième partie (96 p.) qui démontre en trois sous-ensembles les fondements de ce communisme politique qui prendra figure d'archétype fondateur une pratique conspiratrice destinée à assurer la prise de contrôle du pouvoir d'État, préalable à l'instauration de la démocratie, une doctrine de rassemblement par voie de propagande et de recrutement d'affidés principalement "ouvriers", une ambition démiurgique de transformation de l'ordre social et des rapports de propriété et de distribution, ce qui différenciait radicalement ce communisme républicain $d u$ cabétisme des Icariens. Forte et brève conclusion consacrée à faire sentir la cohérence et la pérennité mais aussi les handicaps structurels des héritages de mémoire et de culture qui ont trouvé en ce lieu et en ce temps, une part de leur puissance évocatrice.

3 À côté de beaucoup d'autres vues originales ou récapitulatives, trois apports essentiels m'ont paru caractériser cet ouvrage important. Retenons en premier lieu la démonstration très convaincante qui nous conduit à penser que la tradition, l'utopie communautaire - voire " communiste »- et les formes culturelles nées des pratiques de solidarité paysanne ou corporative, ne sont pas intervenues directement dans le processus positif de construction de l'imaginaire communiste du XIX siècle mais qu'elles se sont ordonnées de manière spécifique en France - surtout à Paris, Lyon et autres cités manufacturières - à travers la reprise en héritage de l'expérience jacobine et la compréhension du rôle de l'État national centralisé, recomposé sous l'effet de la Révolution française. D'où l'expression si suggestive de «communisme unitaire » que Théodore Dezamy voulait donner à sa doctrine. Devient donc centrale dans ce mouvement, l'articulation du politique - détermination des enjeux, luttes pour le pouvoir, formes de la représentation etc. - et du projet social de construction d'une société débarrassée de l'exploitation et des inégalités de là assurément le recours au despotisme de l'État le plus souvent accepté comme instrument nécessaire dans l'accouchement du monde nouveau. Deuxième idée force la référence sociologique dans les « imaginaires sociaux du communisme» (p. 122) instruit des travaux d'Alain Faure et de William Sewell, Alain Maillard souligne fortement le "républicanisme ouvrier " dont la racine demeure si vigoureuse dans l'histoire contemporaine de la France un républicanisme qui s'inscrit dans la «continuité » d'une histoire populaire et révolutionnaire qui a toujours fait la part belle (et la plus héroïque) aux pratiques normées des travailleurs des métiers, surtout urbains vive cette "république des blouses » qui ne sera pas celle des «messieurs » ... Mais son ambition proclamée est de 
libérer tous les hommes du préjugé, de l'ignorance et des dominations. Les femmes également Ici, il faut bien constater qu'au regard des travaux de nombre d'historiennes, notamment de ceux de Michèle Riot-Sarcey, l'analyse de Maillard m'a paru un peu courte, peut-être dépassée. Cette ambition émancipatrice et fraternelle, rapproche plus qu'elle n'éloigne, malgré le "matérialisme» (philosophique) affiché par nombre d'entre eux, «l'imaginaire christique» (p. 145) des communistes de 1840 des penseurs chrétiens contemporains, comme l'avait déjà suggéré Henri Desroche qui évoquait pour sa part le «christ zélote» des babouvistes. Tout cela sur fond de «mystique républicaine " selon le mot de Charles Péguy.

Troisième apport enfin cette belle évocation de la « génération de 1840 » qui construit, contre la doxa libérale et presque dix ans avant les barricades de février et de juin, une " contre-mémoire collective » (p. 163) à la mamelle de laquelle se formeront tous les révolutionnaires français (et souvent européens) dont les exploits comme les enseignements culmineront de la Commune de 1871 jusqu'aux recompositions ou reconstructions consécutives à la révolution de 1917 et aux pratiques, plus diverses qu'on ne le croyait, de la «bolchévisation » ... mais c'est ici une toute autre histoire

Dans cet ensemble pionnier, dans ce livre comme dans celui bien antérieur de GalanteGarrone, deux figures émergent, Théodore Dezamy, «l'anti-Cabet », celui qui mène l'assaut contre "l'insupportable père Cabet» et Jean-Jacques Pillot, l'ancien séminariste devenu si fort théoricien et logicien l'un et l'autre ont su dans une fidélité essentielle, transmettre, élargir et renouveler le grand héritage idéologique issu de la Révolution française et transmis par Buonarroti aux révolutionnaires du xix ${ }^{e}$ siècle. Un événement fait naturellement référence le célèbre "banquet communiste " de Belleville du 1er juillet 1840 dont la notoriété a débordé le cadre de Paris et même de la France (Weitling et des convives polonais). Une structure fondatrice émerge la reprise de l'héritage républicain avec la construction des «sociétés secrètes communistes et ouvrières » qui vont donner une sorte de " modèle » primitif à l'organisation d'un parti politique et de classe, simultanément décentralisé dans l'action de recrutement et de propagande et centralisé dans la formation de son programme et de ses règles d'organisation les historiens de la Révolution française retrouveront ainsi quelques traits de l'ancienne structuration des sociétés populaires (cf. Boutier-Boutry, C. Peyrard, D.Pingué, J. Bernet, etc.).

6 Les quelques indications relevées ne sauraient rendre compte de la richesse d'un livre bien informé, probe dans ses affirmations comme dans ses références, utile comme le sont sous le regard des sociologues historiens, les travaux qui veulent aller au-delà de la superficialité et du clinquant, pour saisir la pérennité et les mutations des héritages culturels durables. Avec Maillard, la course n'a pas été menée le nez dans le guidon. Son livre est riche et vient à point. À lire, assurément. 